\title{
Effects of time of night and sleep stage on perception of sleep in subjects with sleep state misperception
}

\author{
WALLACE B. MENDELSON \\ University of Chicago, Chicago, Illinois
}

\begin{abstract}
Although previous studies have documented that some persons who complain of poor sleep report having been awake when questioned following an awakening from Stage 2 sleep induced by an auditory stimulus, such observations have been confined to the first NREM-REM cycle. We have examined the possibility that the perception of having been awake or asleep may differ across the night. Eight subjects with sleep state misperception were awakened by a $500-\mathrm{Hz}$ tone of progressive amplitude at five time points and were then asked to report whether they believed themselves to have been awake or asleep. This procedure was carried out on an early night during the first sleep cycle, and on a late night during the third sleep cycle, in random sequence. During the early Stage 2 trial, subjects were more likely to report having been awake, whereas in the late Stage 2 trial, they were more likely to report that they had been asleep. Conversely, in the REM sleep trial on the early night, subjects were more likely to report that they had been asleep. On the late night they were more likely to report that they had been awake, a change which was paralleled by a significant drop in scores on the Foulkes Dream Complexity Scale. Calculation of the duration of uninterrupted sleep prior to the trials suggests that this may have been a factor in the Stage 2, but not the REM, findings. There were no significant differences between the early and late REM trials in the frequency of dream reports, quality of dreams, or fragmentation of REM. These data indicate that subjects with sleep state misperception may experience REM sleep very differently in the first and third NREM-REM cycles.
\end{abstract}

One of the most challenging problems in treating patients who complain of poor sleep is dealing with the subgroup who experience sleep state misperception (ASDA, 1991). These individuals describe inadequate quantity or quality of sleep, although polygraphic measures of their sleep are only mildly disturbed. The seminal studies of Rechtschaffen (1968) raised one interesting possibility as to the nature of this disorder; briefly, poor sleepers who were awakened by an auditory stimulus early in NREM sleep tended to report having just been awake, in contrast to good sleepers, who tended to report that they had been asleep. This seemed to suggest that at least one aspect of the poor sleepers' difficulty might be a misperception of their state of consciousness. This finding has been replicated in later studies (Coates et al., 1983; Mendelson, James, Garnett, Sack, \& Rosenthal, 1986; Moore, Bonnet, \& Warm, 1991), which have indicated that administration of the hypnotic medications triazolam (Mendelson, 1993) and zolpidem (Mendelson, 1995a) enhances the likelihood that insomniacs will respond that they have been asleep, but that it will have no effect on normal sleepers (Mendelson, 1995b). One feature of all of these studies, however, has been that the auditory stimuli have been presented dur-

This study was partially supported by NIH Grant 1 K07HL03640 Correspondence and reprint requests should be sent to W. Mendelson, Sleep Research Laboratory, University of Chicago, 5743 S. Drexel Ave., Chicago, IL 60637 (e-mail: wmendels@yoda.bsd.uchicago.edu). ing the first NREM-REM cycle, on which the patients' reports have therefore been based, and it is not clear whether the responses would have been similar if the patients had been tested during subsequent cycles. Although a number of studies have addressed auditory arousal thresholds in normal volunteers across the night (Rechtschaffen, Hauri, \& Zeitlin, 1966; Zimmerman, 1970), minimal data are available on the subjective experience of being awake or asleep in later NREM-REM cycles in normal subjects, and there are no comparable data for insomniacs. For this reason, we are now reporting on a preliminary study of sleep perception in chronic insomniacs who were tested both in the first and in subsequent cycles. Our goal was to determine whether insomniacs would describe their perception of NREM and REM sleep differently in the first and third cycles, and if they did, to develop a theoretical framework that would allow subsequent studies to parse out possible mechanisms that might explain such differences.

\section{METHOD}

There were 8 subjects ( 1 male and 7 female) with a mean age of $32.6 \pm 4.1(S E M)$ years (range, 19-50), who complained of disturbed sleep $4.5 \pm 0.8$ nights per week for $5.5 \pm 0.7$ years. Their self-reports of habitual sleep are found in Table 1. The subjects were seen in the clinic, at which time an interview with the author determined that there was no clinical evidence to suggest psychiatric illness such as major depression or other likely causes of their sleep complaints. With the exception of birth control pills, all subjects were medication free for at least 2 weeks prior to the study. None had a history of alcohol abuse, 
Table 1

Characteristics of Subjects: Subjective Reports of Habitual Sleep (Sleep Habit Questionnaire)

\begin{tabular}{lcc}
\multicolumn{1}{c}{ Measure } & $M$ & $S E M$ \\
\hline Difficulty getting to sleep & 4.0 & $0.5^{*}$ \\
Awakenings during the night & 3.2 & $0.7^{*}$ \\
Early morning awakening & 3.7 & $0.5^{*}$ \\
Daytime sleepiness & 2.4 & $0.6^{*}$ \\
Habitual sleep latency (minutes) & 52.5 & 22.5 \\
Habitual total sleep time (hours) & 4.5 & 0.5 \\
Feels refreshed upon awakening & \multicolumn{2}{c}{ 3 yes, 5 no } \\
\hline
\end{tabular}

*Response on a $1-5$ scale, in which $1=$ not really a problem and $5=a$ very major difficulty.

and all of the subjects agreed to remain alcohol free for the duration of the study. The subjects then underwent a clinical polysomnogram to rule out any other major pathophysiology of sleep (e.g., sleep-disordered breathing, periodic limb movement disorder). When they filled out a morning sleep questionnaire, their estimate of sleep latency was over twice as long as was found on polysomnography (see Table 3), a finding compatible with sleep state misperception.

Subjects then had two experimental nights, which were separated by 1 week, and which occurred in random order. On each experimental night, the subjects were awakened by a $500-\mathrm{Hz}$ electronic tone of progressively increasing amplitude $(2 \mathrm{~dB}$ every $3 \mathrm{sec})$ at five time points across the night. As in our previous studies (Mendelson, 1995a, 1995b), an arousal response was defined as at least $15 \mathrm{sec}$ of alpha EEG activity, or of continuous muscle artifact in all four channels (EEG, two electrooculograms, EMG). If the subject was not awakened by the tone when it reached its maximum of $100 \mathrm{~dB}$, after $1 \mathrm{~min}$ at this amplitude an investigator manually awakened the subject. On one night (which we shall refer to as the early night) these trials occurred during the first NREM-REM cycle (defined as the first occurrence of NREM sleep followed by REM sleep) and were as follows: (1) 5 min after "lights out," at which time subjects have empirically been found to be awake; (2) 10 min after the first sleep spindle; in all studies subjects have been in Stage 2 sleep at this point; (3) $10 \mathrm{~min}$ after the onset of Stage 4 sleep; (4) 5 min after the onset of REM sleep; (5) during the first period of spontaneous waking time after the first REM period (technically this would be during the beginning of the second NREM-REM cycle).

On the "late" experimental night, the same procedures were performed during the third NREM-REM cycle, with the exception of the 5 min after "lights out" time point, which by definition did not occur then. During these trials, an investigator entered the room and asked the subjects a list of questions, including whether (s)he believed that (s)he had been awake or asleep and whether (s)he had been dreaming (Table 2); after each of these questions, the subject was also asked how certain (s)he was of the answer, on a 1-4 scale $(1=$ very uncertain; $4=$ absolutely confident $)$. The Dream Fantasy Scale and Dream Complexity Scale (Foulkes, 1970; Foulkes \& Vogel, 1965) were also administered. The latter assesses both the subject's claim of being awake or asleep as well as the nature of the cognitive content and hence served as a check on the categorical question of whether the subject reported having been awake or asleep. On this scale, scores $\mathbf{I}-6$ are used when a subject claims to have been awake; scores $7-14$ are used when a subject claims to have been asleep. Progressively higher scores represent increasingly complex thought processes and dreaming.

Quantitative data were assessed by two-tailed paired $t$ tests; categorical data were analyzed by two-tailed chi-square tests. Data are reported in terms of the mean \pm the standard error of the mean (SEM).

\section{RESULTS}

\section{Screening Night}

Polygraphic data for the screening sleep recording are found in Table 3. It can be seen that the subjects had moderately disturbed sleep, with a mean sleep efficiency of $80.6 \% \pm 4.0 \%$. Data from the morning sleep questionnaire indicated that they overestimated the time that it took them to fall asleep by a mean of $39 \mathrm{~min}$ and underestimated their total sleep time by 62 min-findings consistent with sleep state misperception.

\section{Perception of Being Awake or Asleep}

Responses to the categorical question "Were you awake or asleep immediately prior to hearing the tone or being called by me?" are found in Table 4 . It can be seen that there were three significant differences between responses between the early and late conditions: (1) During the early Stage 2 awakening, subjects were more likely to report having been awake, whereas in the late awakening, they were more likely to rcport that they had been asleep. (2) During the REM sleep trials on the early night, subjects were more likely to report that they had been asleep; on the late night, they were more likely to report that they had been awake. (3) During the intermittent waking time trials on the early nights, there was a nonsignificant trend $(p<.07)$ for subjects to be more likely to report having been asleep, whereas on the late nights, they were more likely to report having been awake. There were no significant differences in the degree of certainty with which subjects gave their answers at any of these three time points. For the REM trials, for instance, subjects gave certainty scores of $3.1 \pm 0.3$ and $3.6 \pm 0.2$ on the early and late nights, respectively $(1=$ very uncertain; $4=a b$ solutely confident).

Scores on the Dream Complexity Scale during the REM sleep trials dropped from $6.6 \pm 0.8$ (the borderline of a sleep claim) to $3.3 \pm 1.1$ (a waking claim) on the early and late nights, respectively $(p<.03)$. There were no significant changes in the scores at the other time points, though they moved in the directions predicted by the categorical

Table 2

Questions Asked During the Arousal Trials

\footnotetext{
1. Were you awake or asleep immediately prior to hearing the tone or being called by me?

2. How long has it been since I turned off the lights?

3. How long have you been awake since I turned off the lights?

4. What information did you use to decide whether you were awake or asleep?

5 . Were you dreaming?

6 . Were the things going on in your mind more like thoughts or pictures?

7. Were you watching yourself in your mind, or did it seem like you were actually participating in the activities?

8. Do you remember what you were thinking/dreaming about?
}

Note-After each answer the subjects were asked to respond on a 1-4 scale as to how certain he/she was of the answer just given: $1=$ very uncertain and $\mathbf{4}=$ absolutely confident. 
Table 3

Screening Night Sleep Data

\begin{tabular}{lcc}
\multicolumn{1}{c}{ Measure } & $M$ & SEM \\
\hline Total sleep time & 359.5 & 15.7 \\
Sleep latency & 38.6 & 17.2 \\
Sleep efficiency & $80.6 \%$ & $4.0 \%$ \\
REM & 19.4 & 1.7 \\
WASO & 53.9 & 17.8 \\
Subjective total sleep & 297.5 & 30.1 \\
Subjective sleep latency & 77.8 & 18.8 \\
\hline
\end{tabular}

Note-All values except sleep efficiency are given in minutes. Sleep efficiency $=$ total sleep time/total recording time, expressed as a percentage.

question of being awake versus asleep (e.g., scores tended to rise from the early to late night during the Stage 2 trials).

\section{Characterization of Cognitive Material}

There were no significant differences between the early and late night responses to the other questions, including whether cognitive material was more like a thought or a picture and whether the subject had had a sense of viewing or participating. There were also no differences in scores on the Foulkes Dream Fantasy Scale.

\section{Certainty of Responses}

There were no significant differences between the early and late night reports as to the subjects' certainty of their responses to any of the questions.

\section{Auditory Arousal Threshold}

The data on amplitude of tones necessary to induce arousals are found in Table 5. Although with this sample size the differences across sleep stages did not reach statistical significance, the pattern is consistent with most available data (e.g., Rechtschaffen et al., 1966), with highest amplitudes on the early night being in Stage 4, and with Stage 2 and REM sleep having similar values. There were no significant differences between the same stage tested during the early and late nights, although the late night values tended to be lower in the late night Stage 2 and REM as opposed to the early nights. (A comparison of Stage 4 was not possible, because there was not enough Stage 4 sleep late at night to make experimental trials possible.)

\section{CONCLUSIONS}

In summary, $75 \%$ of the subjects awakened by an auditory tone of increasing amplitude in early Stage 2 sleep reported that they had been awake, whereas during Stage 2 sleep in the third NREM-REM cycle, this dropped to $25 \%$. In REM sleep, the converse process occurred: In early REM sleep, most insomniacs believed themselves to have been asleep, whereas in the third REM period, they reported that they had been awake. They tended $(p<.07)$ to report that they had been asleep when questioned after early intermittent waking time, whereas after the third sleep cycle, they tended to report that they had been awake. These observations raise the possibility that subjects with sleep stage misperception may experience the same sleep stage differently in the first and third sleep cycles. Let us examine these findings and consider possible factors that may underlie them.

This finding that during early Stage 2 these subjects tend to report that they have been awake replicates the results of previous studies (Coates et al., 1983; Mendelson et al., 1986; Moore et al., 1991; Rechtschaffen, 1968). The finding that reports of being awake or asleep change in the third sleep cycle could conceivably be due to several factors.

\section{Amount of Preceding Sleep}

Although data do suggest that under certain conditions (Rechtschaffen et al., 1966), but not others (Zepelin, Schmedlen, \& McCallum, 1989; Zimmerman, 1970), auditory arousal threshold drops with accumulated sleep, we are not aware of any data addressing whether accumulated sleep might influence sleep perception. One possible hypothesis, however, might be that in the same stage, greater durations of preceding sleep make a report of one's having been asleep more likely. The experimental design, in which subjects were awakened $10 \mathrm{~min}$ after the first sleep spindle during the early night, dictated that there would be less sleep prior to the forced awakening on that night, and indeed this was confirmed by an analysis of the number of minutes of continuous sleep (amount of sleep time since any awakening lasting at least $3 \mathrm{~min}$ ). As can be seen in Table 6, during the early night there was a mean of $15.1 \mathrm{~min}$ of sleep before the early awakening, as opposed to

Table 4

Responses to the Question "Were You Awake

or Asleep Immediately Prior to Hearing

the Tone or Being Called by Me?"

\begin{tabular}{|c|c|c|c|c|c|}
\hline & \multicolumn{2}{|c|}{ Early } & \multicolumn{2}{|c|}{ Late } & \multirow{2}{*}{$\underset{\left(\chi^{2}\right)}{p}$} \\
\hline & Awake & Asleep & Awake & Asleep & \\
\hline 5 min after "lights out" & 7 & 0 & - & $*$ & \\
\hline 10 min after first spindle & 6 & 2 & 2 & 6 & $<.043^{\ddagger}$ \\
\hline Stage 4 & 1 & 2 & - & $\dagger$ & \\
\hline REM & 1 & 7 & 5 & 2 & $<.02$ \\
\hline Intermittent waking time & 2 & 6 & 5 & 2 & $<.07$ \\
\hline
\end{tabular}

*By definition, there was no situation matching 5 min after "lights out" during the third NREMREM cycle, so this particular trial was not performed. ${ }^{+}$There was insufficient Stage 4 sleep in the third NREM-REM cycle to perform the trials. ‡Not significant by Fisher’s exact probability test. 
Table 5

\begin{tabular}{|c|c|c|c|c|c|}
\hline \multicolumn{6}{|c|}{ Amplitude of Electronic Tone (in Decibels) Used in Awakening Trials } \\
\hline \multirow[b]{2}{*}{ Measure } & \multicolumn{2}{|c|}{ Early } & \multicolumn{2}{|c|}{ Late } & \multirow[b]{2}{*}{$p$} \\
\hline & $M$ & $S E M$ & $M$ & $S E M$ & \\
\hline 5 min after "lights out" & 56.5 & 7.2 & ${ }^{*}$ & & \\
\hline 10 min after first spindle & 64.4 & 7.0 & 59.2 & 10.0 & n.s. \\
\hline Stage 4 & 79.0 & 13.5 & + & & \\
\hline REM & 65.0 & 7.9 & 63.1 & 6.8 & n.s. \\
\hline Intermittent waking time & 60.4 & 10.1 & 57.7 & 9.9 & n.s. \\
\hline
\end{tabular}

*By definition, there was no situation matching 5 min after "lights out" during the third NREM-REM cycle, so this particular trial was not performed. ${ }^{\dagger}$ There was insufficient Stage 4 sleep in the third NREM-REM cycle to perform the trials.

109.2 min of sleep preceding the late awakening $(p<.03)$. Previous data have also indicated that in normal subjects, the frequency of reports of cognitive activity decreases as a function of the time in NREM sleep after the end of an REM period (Goodenough, Lewis, Shapiro, Jaret, \& Sleser, 1965). Thus, the amount of prior sleep may in principle be an explanation for the difference in perception of Stage 2 sleep at the two time points. Interestingly, the converse pattern was seen for intermittent waking time: There was more continuous sleep prior to the early than to the late awakening, which would be consistent with the trend for the insomniacs to report that they had been asleep and awake respectively at those two time points. Interestingly, there was no significant difference in the amount of continuous sleep before the REM awakenings, making this seem unlikely as an explanation of the difference in sleep perception. In terms of the Stage 2 findings, a follow-up study in which the amount of prior sleep is held constant would be necessary in order to confirm that this is the relevant mechanism.

\section{Time of Night}

It is certainly possible that there is a circadian influence on perception of sleep stages, analogous perhaps to reports of a circadian effect on time estimation (Hancock, Vercruyssen, \& Rodenburg, 1992). This preliminary study did not address this issue, because it was designed to determine a more fundamental question: whether there would be differences in subjective reports in the first and third sleep cycles. Given this finding, a next step would be to de- sign a study in which the timing of sleep is scheduled to hold circadian time constant at the awakening points.

\section{Duration of Prior REM Sleep and Amount of Fragmentation of REM Sleep}

One possible explanation for the REM sleep findings is that there was a difference in the amount or continuity of REM sleep immediately prior to the trial. In order to examine this, we calculated the total minutes in the preceding REM sleep period immediately before the trial, and the number of 30-sec epochs of waking during the preceding REM sleep. There was no significant difference in the duration of REM sleep prior to the trials in the early $(6.4 \pm$ $0.3 \mathrm{~min})$ and late $(6.2 \pm 0.7 \mathrm{~min})$ nights. Similarly, there was no significant difference in the number of $30-\mathrm{sec}$ epochs of arousal inside the REM sleep period prior to the trials on the early $(1.2 \pm 0.4)$ and late $(0.6 \pm 0.2)$ nights. Thus, by these measures, there was no evidence that differences in duration or continuity of REM sleep might explain the differences in perception of being awake or asleep.

\section{The Presence of Dreaming}

Another hypothesis that might explain the REM sleep findings is that the experience of dreaming (as reflected in a report of having dreamed) contributes to the perception of one's having been asleep. We found no difference in the frequency of reporting dreams in early versus late REM sleep, although, interestingly, the report frequency in these insomniacs was relatively low (25\%) at both points in comparison with frequencies reported in normal subjects in

Table 6

Amount of Continuous Sleep Prior to Trials

\begin{tabular}{|c|c|c|c|c|c|}
\hline \multirow[b]{2}{*}{ Measure } & \multicolumn{2}{|c|}{ Early } & \multicolumn{2}{|c|}{ Late } & \multirow[b]{2}{*}{$p$} \\
\hline & $M$ & $S E M$ & $M$ & $S E M$ & \\
\hline 5 min after "lights out" & 0 & 0 & $*$ & & \\
\hline 10 min after first spindle & 15.1 & 1.0 & 109.2 & 33.7 & $<.03$ \\
\hline Stage 4 & 42.5 & 5.2 & + & & \\
\hline REM & 43.8 & 9.9 & 45.3 & 8.7 & n.s. \\
\hline Intermittent waking time & 41.1 & 7.5 & 24.8 & 5.7 & $<.02$ \\
\hline
\end{tabular}

Note - Values are in minutes of sleep since the last awakening (defined as at least 3 min of an awake EEG) preceding the trial. *By definition, there was no situation matching 5 min after "lights out" during the third NREM-REM cycle, so this particular trial was not performed. ${ }^{+}$There was insufficient Stage 4 sleep in the third NREM-REM cycle to perform the trials. 
the first REM period. In early REM, 6 subjects reported not dreaming, of whom 5 reported having been asleep; in late REM, 4 subjects reported not dreaming, of whom 4 said they had been awake $\left[\chi^{2}(1)=4.41, p<.04\right]$. A report of having dreamed was not significantly associated with a report of having been asleep, though it seems likely that this reflects the small number of dream reports.

\section{The Quality of Dream Material}

It also seems possible that some qualitative aspect of dreaming differs between early and late REM sleep, contributing to the disparity in reports of one's having been awake or asleep. We found no difference in the frequency of reports of having thought-like or picture-like content, nor of watching versus participating in the cognitive activity across the two points. In summary, the categorical measures that we used did not reveal a difference in the quality of cognitive activity in early versus late REM, though it remains possible that a more elaborate assessment might reveal such differences.

Finally, it should be noted that the methodology and the choice of subjects could affect the response rates regarding one's having been awake or asleep. These include the possible effects of the following:

\section{The "Method of Limits" Versus the "Method of Constant Stimuli"}

In the present study, a tone of progressively greater amplitude was played (the "method of limits"), as opposed to determining the frequency of correct verbal responses to variable numbers of tones of a fixed amplitude which had previously been determined to be at the "approximate awakening thresholds" (Rechtschaffen et al., 1966). It has been argued that the latter method reduces the amount of incorporation of dream material that might influence arousal thresholds. The conclusions drawn from the present study are limited to the methodology of awakening subjects by the method of limits.

\section{Abrupt Versus Gradual Awakenings}

It is also possible that a tone of progressively louder amplitude might produce different results than would a single tone of very high amplitude. Indeed, previous data indicate that abrupt awakenings increase the likelihood that REM reports will include dreaming and that NREM reports will yield no content (Goodenough et al., 1965); phrased differently, in both REM and NREM sleep, gradual awakenings enhance the likelihood of reports of "thinking." Similarly, gradual awakenings from NREM sleep have been reported to produce thought content that is less bizarre and emotional (Starker \& Goodenough, 1970). It is possible that the gradually increasing tone used in the present study thus contributed to the relatively low rate of dream reports in REM sleep. On the other hand, since the same technique was used on both nights, this does not explain the difference in perception of having been awake or asleep in early or late REM sleep.

\section{Number of Prior Forced Arousals}

The number of previous trials on a given night has been reported to influence thresholds on later trials in normal subjects (Zepelin et al., 1989), and it remains possible that the number of prior trials also influences the perception of having been awake or asleep in insomniacs. In the present design, there was a small difference in the number of preceding trials on the two nights, since (by definition) on the late night we did not have a trial 5 min after "lights out." In future follow-up studies, this question could be addressed in the following manner: Trials could be performed on multiple nights (rather than the two nights done in this original study), with the number of prior trials held constant.

\section{Insomniacs Versus Normals}

Finally, it should be noted that this study was performed on insomniacs. This choice of subjects is of relevance to several aspects of the data, including the relatively small amount of Stage 4 sleep (which allowed us to do only three Stage 4 trials during the early night) as well as the responses to questions about having been awake or asleep. Previous studies have indicated that insomniacs differ in their report of having been awake or asleep following trials in early Stage $2(1,4,5)$, and it is possible that the differences between perception of being awake or asleep in early versus late awakenings reported here would not be seen in normal sleepers. This study seems to suggest, though, that insomniacs, when tested with this methodology, experience sleep in the third NREM-REM cycle as being different from that in the first cycle. A common clinical experience is that an insomniac complains that (s)he had great difficulty getting to sleep and then late at night did fall asleep only to be awakened by the alarm clock. The present data lead to the speculation that in a subgroup of such patients, the explanation may be that their subjective perception of sleep in the early morning hours differs from that at the beginning of the sleep period.

\section{REFERENCES}

AMERICAN SleEP Disorders Association (1991). International classification of sleep disorders: Diagnostic and coding manual (1991). Rochester, MN: Author.

Coates, T. J., Killen, J. D., Silberman, S., Marchini, J., Hamilton, S., \& Thoresen, C. E. (1983). Cognitive activity, sleep disturbance, and stage specific differences between recorded and reported sleep. Psychopharmacology, 20, 243.

Foulkes, D. (1970). The psychology of sleep. New York: Scribner's.

Foulkes, D., \& VoGel, G. (1965). Mental activity at sleep onset. Journal of Abnormal Psychology, 70, 231-243.

Goodenough, D. R., Lewis, H. B., Shapiro, A., Jaret, L., \& Sleser, I. (1965). Dream reporting following abrupt and gradual awakenings from different times of sleep. Journal of Personality \& Social Psychology, 2, 170-179.

Hancock, P. A., Vercruyssen, M., \& Rodenburg, G. H. (1992). The effect of gender and time-of-day on time perception and mental workload. Current Psychology: Research and Reviews, 11, 203-225.

Mendelson, W. B. (1993). Pharmacologic alteration of the perception of being awake or asleep. Sleep, 16, 641-646.

MENDELSON, W. B. (1995a). Effects of flurazepam and zolpidem on the perception of sleep in insomniacs. Sleep, 18, 92-96. 
MEndelson, W. B. (1995b). Effects of flurazepam and zolpidem on the perception of sleep in normal volunteers. Sleep, 18, 88-91.

Mendelson, W. B., James, S. P., Garnett, D., Sack, D. A., \& RosenTHAL, N. E. (1986). A psychophysiological study of insomnia. Psychiatry Research, 19, 267-284.

MoORE, S. E., BonNeT, M. H., \& WARM, J. S. (1991). Estimates of sleep latency in the morning and at sleep onset in insomniac and normal subjects. Sleep Research, 10, 219.

Rechtschaffen, A. (1968). Polygraphic aspects of insomnia. In H. Gastaut, E. Lugaresi, T. B. Cerone, \& G. Coccagna (Eds.), The $a b-$ normalities of sleep in man (pp. 109-118). Bologna: Gaggi.

Rechtschaffen, A., Hauri, P., \& Zeitlin, M. (1966). Auditory awakening thresholds in REM and NREM sleep stages. Perceptual \& Motor Skills, 22, 927-942.
Starker, S., \& GoOdenough, D. R. (1970). Effects of sleep state and method of awakening upon thematic apperception test productions at arousal. Journal of Nervous \& Mental Disease, 150, 188-194.

ZePelin, H., Schmedlen, L. K., \& McCallum, C. S. (1989). Withinnight variation of the auditory awakening threshold: The role of procedural effects. Sleep Research, 18, 130.

ZimMERMAN, W. B. (1970). Sleep mentation and auditory awakening thresholds. Psychophysiology, 6, 540-549.

(Manuscript received August 25, 1997; revision accepted for publication January 21, 1998.) 